\title{
PERFIL DOS LAUDOS DE EXAMES RADIOGRÁFICOS INTRABUCAIS REALIZADOS NO SUL CATARINENSE
}

\section{PROFILE OF REPORTS OF INTRAORAL RADIOGRAPHS PERFORMED IN THE SOUTH OF SANTA CATARINA STATE}

\author{
Karoline Rossi* \\ Luciane Bisognin Ceretta" \\ Priscyla Waleska Simões"** \\ Renan Antônio Ceretta**** \\ Patricia Fernandes Avila Ribeiro
}

\begin{abstract}
RESUMO
Introdução: O exame radiográfico pode ser essencial para o diagnóstico das doenças bucais assintomáticas e na confirmação de sinais e sintomas clínicos. Buscou-se verificar o perfil epidemiológico dos pacientes, do Sistema Único de Saúde do município de Criciúma/SC, submetidos a exames radiográficos intrabucais, realizados por uma clínica de radiologia odontológica particular. Objetivo: Buscou-se caracterizar os pacientes atendidos pelo Sistema Único de Saúde em um município Sul Catarinense, e submetidos a exames radiográficos intrabucais. Método: Foi realizado um estudo transversal, descritivo, com abordagem quantitativa, com uma amostra de 576 radiografias intrabucais, distribuídas em 290 laudos, no período de um ano. Resultados: A cárie $(61,5 \%)$ foi a doença mais frequente e, entre os achados radiográficos, as cristas alveolares normais as mais prevalentes $(43,7 \%)$. A doença cárie foi significativamente associada ao gênero $(p=0,001)$, sendo mais frequente no feminino (58,7\%). Houve associação estatisticamente significativa da idade dicotomizada em relação a cárie e as cristas alveolares normais (ambos com $p<0,001$ ), mais frequentes em indivíduos mais jovens. Conclusões: O perfil predominante foi composto por mulheres adultas de meia idade, a cárie foi a mais frequente entre as lesões e as cristas alveolares entre os achados radiográficos.
\end{abstract}

Descritores: Epidemiologia • Radiografia • Diagnóstico.

\section{ABSTRACT}

Introduction: Radiographic examination may be essential for the diagnosis of asymptomatic oral diseases and confirmation of clinical signs and symptoms. It was attempted to verify the epidemiological profile of patients, the Health System in the city of Criciuma/SC, submitted to intra-oral radiographic examinations by a private dental radiology clinic. Objective: It was attempted to verify the patients attended in the Sistema Único de Saúde in the South of Santa Catarina state, submited to intra-oral radiographs. Methods: It was conducted a cross-sectional descriptive study with a quantitative approach, from a sample of 576 intra-oral radiographs, distributed in 290 reports, within one year. Results: Caries (61.5\%) were the most common disease and among the radiographic findings, normal alveolar ridges (43.7\%). The caries was significantly associated with gender $(p=0.001)$, being more frequent in females $(58.7 \%)$. There was a statistically significant association between dichotomized age in relation to decay and the normal alveolar ridges (both $p<0.001$ ), more common in younger individuals. Conclusions: The profile was composed of adult middle-aged women, caries were the most frequent among the injury and the alveolar ridges among the radiographic findings.

Descriptors: Epidemiology • Radiography • Diagnosis.

* Graduanda em Odontologia - Universidade do Extremo Sul Catarinense - Email: karoline_rossi@hotmail.com.

** Doutora em Ciências da Saúde - Universidade do Extremo Sul Catarinense. Professora do Curso de Odontologia e Professora Pesquisadora no Programa de Pós-Graduação em Saúde Coletiva - Universidade do Extremo Sul Catarinense. Email: luk@unesc.net.

*** Doutora em Ciências da Saúde - Universidade do Extremo Sul Catarinense. Professora do Curso de Odontologia e Professora Pesquisadora no Programa de Pós-Graduação em Saúde Coletiva - Universidade do Extremo Sul Catarinense. Email: pri@unesc.net.

**** Doutor em Ciências da Saúde - Universidade do Extremo Sul Catarinense. Professor do Curso de Odontologia - Universidade do Extremo Sul Catarinense. Email: rce@unesc.net.

***** Mestranda em Patologia Bucal. Professora do Curso de Odontologia - Universidade do Extremo Sul Catarinense. Email: pavila@unesc. net. 


\section{N T R O D U Ç Ã O}

Desde a descoberta dos Raios X por Wilhelm Conrad Röntgen, em 1895, os exames radiográficos têm passado por grande progresso na área da Odontologia e seu uso tem sido cada vez mais importante para elaboração de diagnóstico. As técnicas de imagem, usadas em odontologia, podem ser classificadas como: intrabucal e extrabucal, analógico e digital ${ }^{1}$.

Um estudo radiográfico longitudinal nos Estados Unidos revelou que 13\% de todos os procedimentos e cerca de $42 \%$ de todos os exames para a elaboração do diagnóstico são radiografias ${ }^{2}$. O exame radiográfico é de fundamental importância para o diagnóstico das doenças bucais, muitas vezes assintomáticas, e na confirmação de sinais e sintomas de doenças verificadas clinicamente ${ }^{3}$.

A indicação de exames radiográficos em Odontologia deve ser precedida por uma correta anamnese e um criterioso exame clínico. O cirurgião-dentista deve conhecer os princípios de formação da imagem, os efeitos biológicos da radiação, técnicas radiográficas, métodos de processamento e interpretação da imagem obtida. Dessa forma, é possível fazer a correta seleção da técnica radiográfica a ser empregada, evitando exposição à radiação desnecessária. As técnicas radiográficas intrabucais, normalmente, são a primeira opção de escolha, pois apresentam maior detalhe, são mais acessíveis para o cirurgião-dentista e proporcionam menor dose de radiação aos pacientes ${ }^{4,5,6}$.

Diante dessa perspectiva, esta pesquisa tem por objetivo verificar o perfil dos pacientes atendidos pelo Sistema Único de Saúde em um município Sul Catarinense, submetidos a exames radiográficos intrabucais.

\section{MÉ T O D O}

Foi realizado um estudo transversal, descritivo, retrospectivo, de abordagem quantitativa, aprovado pelo Comitê de Ética do local onde foi realizada a pesquisa, sob o protocolo 995.028/2015.

A população deste estudo foi composta por pacientes do Sistema Único de Saúde (SUS) do município de Criciúma - SC sub- metidos a tomadas radiográficas intrabucais em uma clínica de radiologia odontológica, no período de julho de 2013 a julho de 2014, para o complemento de diagnóstico dos cirurgiões-dentistas da rede. Foi estimada uma amostra de 576 radiografias intrabucais, distribuídas em 290 laudos.

Os dados foram coletados de laudos das radiografias selecionadas na amostra e foram separados de acordo com cada grupo de radiografia e regiões dentárias neles presentes, mediante a aprovação do local da realização da pesquisa. Foram excluídos da amostra os laudos que apresentaram mais de uma hipótese diagnóstica.

As variáveis voltaram-se para a presença, quantidade e tipo de cada doença e achado radiográfico. As variáveis independentes foram gênero e idade. Os achados radiográficos investigados foram: cálculos salivares, cristas alveolares normais, tratamento endodôntico, extrusão dentária, ausência dentária, dentes inclusos, dentes impactados, remanescentes radiculares, desmineralização, destruição coronária, dentes em formação, dentes em estágio de esfoliação, fusão dentária, dilaceração, rizólise, raiz supranumerária, dentes em processo de erupção, alvéolo em cicatrização, agenesia e dentes em posição ectópica.

As lesões investigadas foram: cárie, periapicopatia, fratura óssea, fratura dentária, reabsorção alveolar horizontal, reabsorção alveolar vertical, reabsorção radicular externa, reabsorção radicular interna, lesão endopério, cisto paradentário, lesão de furca e cálculo de glândula salivar (sialolito).

Após a coleta de dados foi elaborado um banco de dados no software Statistical Package for the Social Sciences (SPSS) versão 22, aplicativo também utilizado para a análise estatística. Foi avaliada a normalidade da idade, quantidade de lesões e de achados radiográficos pelo teste de Kolmogorov-Smirnov, que revelou distribuição não gaussiana para essas variáveis; assim, foram calculados a mediana e o intervalo interquartil. Também foi estimada a frequência absoluta (n) e relativa (\%) para as variáveis qualitativas.

Foi utilizado o teste de Qui-quadrado
ROSSI K

CERETTA LB

SIMÕES PW

CERETTA RA

RIBEIRO PFA

perfil dos lau -

DOS DE EXAMES

RADIOGRÁFICOS

INTRABUCAIS

REALIZADOS NO

SUL CATARINEN-

$S E$

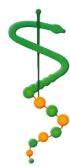

REV, ODONTOL.

Univ. CID. São

PAULO

2017; 29(2):

$110-7$, MAI - AGO 
ROSSI K

CERETTA LB

SIMÕES PW

CERETTA RA

RIBEIRO PFA

PERFIL DOS LAUDOS DE EXAMES RADIOGRÁFICOS INTRABUCAIS REALIZADOS NO SUL CATARINENSE

REV. ODONTOL. UNIV, CID . SÃO

PAULO 2017; 29(2): 110 - 7, MAI - AGO de Pearson para quantificar a associação ou independência entre a idade dicotomizada pela mediana e as demais variáveis categóricas. O mesmo teste também foi utilizado para avaliar a associação ou independência entre o gênero e a presença de lesão, de achados e tipo de radiografia; entre o gênero, e os achados e lesões; e entre a idade dicotomizada pela mediana, e os achados e lesões.

O Teste $U$ de Mann-Whitney foi utilizado para comparar a quantidade de lesões, de achados radiográficos e a idade em relação ao gênero. Para todos os testes supracitados foi considerado um nível de significância $\alpha=0,05$ e intervalo de confiança de 95\%.

\section{RESULTADOS}

O estudo contemplou uma amostra de 290 laudos radiográficos correspondendo a 576 radiografias. Considerando a análise realizada a partir dos laudos radiográfi$\cos (n=290)$, a mediana de idade observada foi de 30 anos $(15,75-44,0)$. Em relação ao gênero, a maioria dos pacientes foi do gênero feminino $(56,6 \%$; $n=164)$. A presença e a quantidade das lesões e os achados radiográficos apresentados nos laudos estão descritos na Tabela 1.

A Tabela 2 ilustra os achados radiográficos e as lesões mais frequentes, analisados por região dentária, que foram mencionadas nos laudos. Dentre as lesões, a cárie $(61,5 \%$; $n=601)$ e a reabsorção alveolar horizontal $(22,3 \% ; n=218)$ foram as mais predominantes. Referente aos achados radiográficos, os mais prevalentes foram as cristas alveolares normais (43,7\%; $\mathrm{n}=286)$ e os cálculos salivares proximais $(20,7 \% ; n=136)$.

$\mathrm{Na}$ análise dos laudos, achados radiográficos, tipo de radiografia e idade, embora os resultados possam sugerir associação do gênero com a presença de lesões, não houve significância estatística, o que pode ser observado na Tabela 3.

Observamos associação estatisticamente significativa entre algumas lesões e o gênero dos pacientes, destacando-se a cárie $(p=0,001)$ e lesões do periápice $(p=0,006)$. Assim, a cárie foi mais frequen-

Tabela 1 - Características gerais dos laudos radiográficos - Criciúma, SC, Brasil - jul. 2013 a jul. 2014.

\begin{tabular}{lc}
\hline \hline Variáveis & $\mathrm{n}(\%)$ \\
\hline Idade mediana (Intervalo interquartil) & $30,00(15,75-44,00)$ \\
Gênero & $120(41,4)$ \\
Feminino & \\
Masculino & \\
Radiografias intrabucais & $89(30,7)$ \\
Interproximal & $197(67,9)$ \\
Periapical & $4(1,4)$ \\
Oclusal & \\
Presença de lesão & $254(87,6)$ \\
Sim & $6(12,4)$ \\
Não & $3,00(2,00-5,00)$ \\
Mediana de lesões (Intervalo Interquartil) & \\
Presença de achados radiográficos & $48(85,5)$ \\
Sim & $42(14,5)$ \\
Não & $2,00(1,00-5,00)$ \\
Mediana de achados radiográficos (Intervalo Interquartil)
\end{tabular}


Tabela 2 - Características gerais das lesões e achados radiográficos da amostra - Criciúma, SC, Brasil - jul. 2013 a jul. 2014.

\begin{tabular}{lc}
\hline \hline Variáveis & $\mathrm{n}(\%)$ \\
\hline Tipos de Lesões ( $\mathrm{n}=978)$ & $601(61,5)$ \\
Cárie & $146(14,9)$ \\
Periapicopatia & $218(22,3)$ \\
Reabsorção alveolar horizontal & $5(0,5)$ \\
Lesão de furca & $8(0,8)$ \\
Outros & \\
Tipos de achados radiográficos ( $\mathrm{n}=656)$ & $136(20,7)$ \\
Cálculos salivares proximais & $286(43,7)$ \\
Cristas alveolares normais & $56(8,5)$ \\
Tratamento endodôntico & $25(3,8)$ \\
Ausência dentária & $26(4,0)$ \\
Dentes impactados & $58(8,8)$ \\
Remanescentes radiculares & $25(3,8)$ \\
Dentes em formação & $44(6,7)$ \\
Outros & \\
\hline \hline
\end{tabular}

Tabela 3 - Características das lesões, achados radiográficos, tipo de radiografia e idade estratificada por gênero por laudo - Criciúma, SC, Brasil - jul. 2013 a jul. 2014.

\begin{tabular}{llll}
\hline \hline Variáveis & $\begin{array}{l}\text { Feminino } \mathrm{n}(\%) \\
\mathrm{n}=164\end{array}$ & $\begin{array}{l}\text { Masculino } \mathrm{n}(\%) \\
\mathrm{n}=120\end{array}$ & $\mathrm{P}$ \\
\hline $\begin{array}{l}\text { Idade mediana } \\
\text { Presença de Lesão }\end{array}$ & $141(86,0)$ & $108(90,0)$ & 0,739 \\
Sim & $23(14,0)$ & $12(10,0)$ & 0,308 \\
Não & $3,00(2,00-5,0)$ & $3,00(2,0-5,0)$ & 0,855 \\
Mediana de lesões (intervalo interquartil) & & \\
$\begin{array}{l}\text { Tipo de Radiografia } \\
\text { Interproximal }\end{array}$ & $59(36,0)$ & $28(23,3)$ & 0,073 \\
$\begin{array}{l}\text { Periapical } \\
\text { Oclusal }\end{array}$ & $103(62,8)$ & $90(75,0)$ & \\
Presença de achados & $2(1,2)$ & $2(1,7)$ & \\
radiográficos & & & \\
Sim & $136(82,9)$ & $107(89,2)$ & 0,139 \\
Não & $28(17,1)$ & $13(10,8)$ & \\
Mediana de achados radiográficos (inter- & $2,00(1,0-4,0)$ & $2,0(1,0-3,0)$ & 0,858 \\
valo interquartil) & & & \\
\hline \hline
\end{tabular}

te no gênero feminino $(58,7 \% ; n=349)$ e as lesões do periápice, no masculino $(54,8 \% ; n=80)$.

No grupo de achados radiográficos, as cristas alveolares normais apresentaram associação ao gênero, considerada estatisticamente significativa $(p=0,015)$, apresentando-se mais frequente em indivíduos do gênero feminino, $(60,4 \% ; n=171)$, e os remanescentes radiculares foram mais en- contrados em indivíduos do gênero masculino $(58,6 \% ; n=34)$, considerando-se associação estatisticamente significativa $(p=0,019)$, conforme mostra a Tabela 4.

A Tabela 5 apresenta as características dos achados radiográficos e lesões de acordo com a idade dicotomizada pela mediana. Foi observada associação entre a idade e a cárie, estatisticamente significativa $(p<0,001)$; assim, observa-se
ROSSI K

CERETTA LB

SIMÕES PW

CERETTA RA

RIBEIRO PFA

PERFIL DOS LAU-

DOS DE EXAMES

RADIOGRÁFICOS

INTRABUCAIS

REALIZADOS NO

SUL CATARINEN-

$S E$
REV, ODONTOL.

UNIV, CID, SÃO

PAULO

2017; $29(2)$ :

$110-7$, MAI - AGO 
I SSN 1983-5183

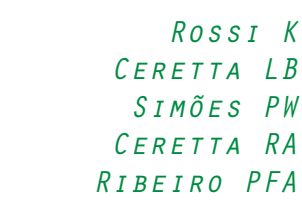

PERFIL DOS LAUDOS DE EXAMES RADIOGRÁFICOS INTRABUCAIS REALIZADOS NO SUL CATARINENSE

Tabela 4 - Características dos achados radiográficos e lesões estratificadas por gênero - Criciúma, SC, Brasil - jul. 2013 a jul. 2014.

\begin{tabular}{|c|c|c|c|}
\hline Variável & Feminino $\mathrm{n}(\%)$ & Masculino $\mathrm{n}(\%)$ & $\mathbf{P}$ \\
\hline \multicolumn{4}{|l|}{ Lesões } \\
\hline Cárie & $349(58,7)$ & $246(41,3)$ & $0,001^{*}$ \\
\hline Periapicopatia & $66(45,2)$ & $80(54,8)$ & $0,006^{*}$ \\
\hline $\begin{array}{l}\text { Reabsorção alveolar } \\
\text { horizontal }\end{array}$ & $106(50,0)$ & $106(50,0)$ & 0,061 \\
\hline Lesão de furca & $2(40,0)$ & $3(60,0)$ & 0,486 \\
\hline \multicolumn{4}{|c|}{ Achados radiográficos } \\
\hline Cálculos salivares & $65(49,6)$ & $66(50,4)$ & 0,098 \\
\hline $\begin{array}{l}\text { Cristas alveolares } \\
\text { normais }\end{array}$ & $171(60,4)$ & $112(39,6)$ & $0,015^{*}$ \\
\hline $\begin{array}{l}\text { Tratamento endodôn- } \\
\text { tico }\end{array}$ & $33(58,9)$ & $23(41,1)$ & 0,612 \\
\hline Ausência dentária & $18(81,8)$ & $4(18,2)$ & $0,012^{*}$ \\
\hline Dentes impactados & $15(57,7)$ & $11(42,3)$ & 0,839 \\
\hline $\begin{array}{l}\text { Remanescentes radi- } \\
\text { culares }\end{array}$ & $24(41,4)$ & $34(58,6)$ & $0,019 *$ \\
\hline Dentes em formação & $14(66,7)$ & $7(33,3)$ & 0,304 \\
\hline
\end{tabular}

*Estatisticamente significativo

Tabela 5 - Associação entre a idade e os tipos de lesões e achados radiográficos - Criciúma, SC, Brasil - jul. 2013 a jul. 2014.

\begin{tabular}{lccc}
\hline \hline & \multicolumn{3}{c}{ Idade } \\
Variáveis & $\begin{array}{c}<\text { mediana } \\
\mathbf{n ( \% )}\end{array}$ & $\begin{array}{c}>\text { mediana } \\
\mathbf{n ( \% )}\end{array}$ & $\mathbf{P}$ \\
\hline Lesão & & & \\
Cárie & $394(65,6)$ & $207(34,4)$ & $<0,001^{*}$ \\
Periapicopatia & $56(38,4)$ & $90(61,6)$ & $<0,001^{*}$ \\
Reabsorção alveolar horizontal & $9(4,1)$ & $209(95,9)$ & $<0,001^{*}$ \\
Lesão de furca & $0(0,0)$ & $5(100,0)$ & $0,015^{*}$ \\
Achados radiográficos & & & \\
Cálculos Salivares & $31(22,8)$ & $105(77,2)$ & $<0,001^{*}$ \\
Cristas alveolares normais & $233(81,5)$ & $53(18,5)$ & $<0,001^{*}$ \\
Tratamento endodôntico & $13(23,2)$ & $43(76,8)$ & $<0,001^{*}$ \\
Ausência dentária & $10(40,0)$ & $15(60,0)$ & 0,143 \\
Dentes impactados & $22(84,6)$ & $4(15,4)$ & $0,001^{*}$ \\
Remanescentes radiculares & $10(17,2)$ & $48(82,8)$ & $<0,001^{*}$ \\
Dentes em formação & $25(100,0)$ & $0(0,0)$ & $<0,001^{*}$ \\
\hline \hline
\end{tabular}

*Estatisticamente significativo

também na referida tabela que a cárie foi predominante em indivíduos mais jovens, ou seja, com menos de 31 anos (65,6\%; $\mathrm{n}=394)$; no entanto, a reabsorção alveolar horizontal que também apresentou associação estatisticamente significativa à idade $(p<=0,001)$ foi mais encontrada em pacientes com idade maior que a mediana (95,9\%; $n=209)$.

Considerando os achados radiográficos, os cálculos salivares e cristas alveolares normais apresentaram associação estatisticamente significativa $(p<0,001)$ com a idade; assim, pode-se observar também 
que os cálculos salivares e as cristas alveolares foram mais frequentes em indivíduos mais velhos.

\section{I SCUSSÃO}

O uso de exames complementares tem sido um instrumento na elaboração do diagnóstico perante as enfermidades bucais, entre eles o uso de radiografias intrabucais ${ }^{7}$. Segundo o Ministério da Saúde, a doença cárie continua sendo a lesão bucal que mais acomete a população brasileira, no entanto, considerando-se dados da última pesquisa de saúde bucal da doença, houve um declínio da mesma, de $19 \%$ na idade dos 12 anos $^{8}$. Para que se possa fazer a prevenção e o tratamento das lesões cariosas, existem vários métodos de diagnóstico disponíveis, entre eles o exame clínico e o uso de radiografias intrabucais ${ }^{9}$.

Nosso estudo mostrou que a doença cárie foi a mais frequente dentre as lesões, significativamente associada ao gênero e idade, predominante em mulheres e indivíduos com idade menor que 31 anos. Segundo os resultados obtidos na literatura, no que diz respeito à idade, as crianças estão em um grupo de risco maior, devido à recém-erupção dos dentes. Em relação ao gênero, as mulheres estão em desvantagem, devido a fatores genéticos, hormonais e culturais. Porém, ainda há falta de evidências que provem a relação da doença ao gênero ${ }^{10}$.

A reabsorção do osso alveolar é um dos defeitos ósseos ou sequelas frequentemente causados pela doença periodontal. Para o seu diagnóstico e possível tratamento, é necessário um exame clínico minucioso combinado a radiografias de qualidade. As radiografias fornecem informações essenciais para verificação da reabsorção óssea alveolar e contribuição na determinação do prognóstico e na elaboração do plano de tratamento ${ }^{11}$.

A reabsorção alveolar horizontal ocorreu em $22,3 \%$ das lesões analisadas, com associação estatisticamente significativa em relação à idade, sendo também mais frequente em indivíduos com mais de 31 anos. Quanto ao gênero, não associação significativa. Um estudo que avaliou a perda óssea relatou que, apesar de não haver significância estatística relacionada ao gênero, os indivíduos do gênero masculino têm maior chance de desenvolvimento da doença. Tal característica poderia ser explicada pelos homens possuírem hábitos mais nocivos em relação à cavidade oral, má higiene com elevado índice de placa bacteriana, além do uso de tabaco ${ }^{12}$.

Já é bem estabelecido na literatura que a presença de cálculos salivares está associada com a doença periodontal, representando um fator determinante para que ocorra a doença ${ }^{13}$. Nos resultados de nossa casuística foi observada uma alta frequência de cálculos salivares proximais $(20,7 \%)$ entre os achados radiográficos, sendo mais frequentes em indivíduos mais velhos. Tal característica pode ser relacionada com a proporção de ocorrência da reabsorção alveolar horizontal entre as lesões, também observada em indivíduos mais velhos.

Uma pesquisa mostrou que $92 \%$ das lesões nos maxilares são radiolúcidas e, entre elas, $85 \%$ se apresentam no periápice $^{14}$. Na análise dos laudos radiográficos de nosso estudo, as alterações do periápice representaram 14,9\% das lesões encontradas, sendo significativamente associadas ao gênero e mais frequente no masculino com mais de 31 anos de idade. Nesse contexto, um estudo realizado na Universidade do Rio de Janeiro avaliou a prevalência de periapicopatia em uma população urbana brasileira. Os resultados, mostraram predominância de lesões periapicais no gênero feminino, na faixa etária de 30 a 49 anos de idade, o que se diferencia de nossos achados, ou seja, mais lesões encontradas no gênero masculino $^{15}$.

A presença de dentes impactados não foi um dos achados mais frequentes na nossa pesquisa. Porém, observamos em nossa amostra predominância entre indivíduos mais jovens e do gênero feminino. Tais resultados corroboram um trabalho que avaliou a ocorrência de dentes impactados associados aos sintomas, complicações e tratamento, concluindo que os dentes impactados são encontrados em indivíduos jovens ${ }^{16}$.

Um estudo realizado no Brasil, a respeito do número médio de dentes ausen-
ROSSI K

CERETTA LB

SIMÕES PW

CERETTA RA

RIBEIRO PFA

PERFIL DOS LAU

DOS DE EXAMES

RADIOGRÁFICOS

INTRABUCAIS

REALIZADOS NO

SUL CATARINEN-

$S E$

$\therefore 115$

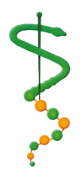

REV, ODONTOL.

UNIV, CID, SÃO

PAULO

2017; $29(2)$ :

$110-7, M A I-A G 0$ 
ROSSI K

CERETTA LB

SIMÕES PW

CERETTA RA

RIBEIRO PFA

PERFIL DOS LAU-

DOS DE EXAMES

RADIOGRÁFICOS

INTRABUCAIS RE-

ALIZADOS NO SUL

CATARINENSE

116 tes entre pacientes adultos, idosos e adolescentes, observou que a perda dentária em idosos acomete principalmente aqueles que possuem menor renda e baixa escolaridade, afetando um maior índice no gênero feminino ${ }^{17}$. Esse estudo obteve resultado semelhante aos da nossa pesquisa, na qual a perda dentária predominou em indivíduos mais velhos e no gênero feminino. Apesar de termos considerado somente laudos radiográficos, tal resultado poderia ser explicado pelo maior acesso de pacientes mais jovens a programas preventivos e água fluoretada, contribuindo para uma menor perda dentária nessa faixa etária.

Embora os resultados do estudo sejam importantes, estes se restringem à análise de laudos radiográficos, sem a realização de um exame clínico prévio para confirmação do diagnóstico.

\section{CONCLUSÕES}

A partir dos dados obtidos apresentamos o perfil dos pacientes do Sistema Único de Saúde de um município Sul Catarinense. A doença cárie destacou-se como a lesão mais prevalente em mulheres mais jovens, seguida pelas lesões de reabsorção alveolar horizontal e periapicopatias. Dentre os achados radiográficos, as cristas alveolares normais e cálculos salivares proximais foram os mais prevalentes. Foi possível evidenciar, também, que o grupo da população foi composto por mulheres adultas de meia idade. 
1. Shah N, Bansal N, Logani A. Recent advances in imaging technologies in dentistry. World journal of radiology 2014 Oct 28;6(10):794-807.

2. Gilbert GH, Weems RA, Shelton BJ. Incidence of dental radiographic procedures during a 48-month population-based study of dentate adults. Oral surgery, oral medicine, oral pathology, oral radiology, and endodontics 2003 Aug;96(2):243-9.

3. Oliveira MV, Silva MBF, Junqueira JLC, Oliveira LB. Avaliação sobre o conhecimento dos cirurgiões-dentistas de Montes Claros-MG sobre técnicas radiográficas, medidas de radioproteção e de biossegurança. Arq Odontol, Belo Horizonte 2012 abr./jun. ;48(2):82-8.

4. Martinez Beneyto $Y$, Alcaraz Banos M, Perez Lajarin L, Rushton VE. Clinical justification of dental radiology in adult patients: a review of the literature. Medicina oral, patología oral y cirugía bucal 2007 May 01;12(3):E244-51í

5. Li G. Patient radiation dose and protection from cone-beam computed tomography. Imaging science in dentistry 2013 Jun;43(2):63-9.

6. Sannomiya EK, Imoto RS, Kawabata CM, Yamamoto MS, Hordiuche RH, Silva RA. Avaliação do emprego dos exames radiográficos e proteção radiológica no cotidiano clínico do cirurgião-dentista na cidade de São Paulo. Rev Fac Odontol Lins 2004 jul.-dez.;16(2):39-43.

7. Moura LB, Blasco MAP, Damian MF. Exames radiográficos solicitados no atendimento inicial de pacientes em uma Faculdade de Odontologia brasileira. Rev odontol UNESP 2014 jul;43(4):252-7.

8. Brasil. Ministério da Saúde. Secretaria de Atenção à Saúde. Secretaria de Vigilância em Saúde. SB Brasil 2010: pesquisa nacional de saúde bucal. Brasília: Ministério da Saúde; 2012.
9. Bader JD, Shugars DA, Bonito AJ Systematic reviews of selected dental caries diagnostic and management methods. Journal of dental education 2001 Oct;65(10):960-8.

10. Martinez-Mier EA, Zandona AF. The impact of gender on caries prevalence and risk assessment. Dental clinics of North America 2013 Apr;57(2):30115.

11. Deepa C, Ramesh AV, Dwarakanath CD, Gayathri G. Interproximal bone loss assessment: comparison of conventional and digital radiographs. IJCD 2012 3(3):23-7.

12. Ashwinirani SR, Suragimath G, Jaishankar HP, Kulkarni P, Bijjaragi SC, Sangle VA. Comparison of diagnostic accuracy of conventional intraoral periapical and direct digital radiographs in detecting interdental bone loss. Journal of clinical and diagnostic research : JCDR 2015 Feb;9(2):Zc35-8.

13. Roberts-Harry EA, Clerehugh V. Subgingival calculus: where are we now? A comparative review. Journal of dentistry 2000 Feb;28(2):93-102.

14. Antoniazzi MCC, Carvalho PL, Koide $\mathrm{CH}$. Importância do conhecimento da anatomia radiográfica para a interpretação de patologias ósseas. Revista Gaúcha de Odontologia 2009 56(2):

15. Berlinck T, Tinoco JM, Carvalho FL, Sassone LM, Tinoco EM. Epidemiological evaluation of apical periodontitis prevalence in an urban Brazilian population. Brazilian oral research 2015 29(51.

16. Msagati F, Simon EN, Owibingire S. Pattern of occurrence and treatment of impacted teeth at the Muhimbili National Hospital, Dar es Salaam, Tanzania. BMC oral health 2013 Aug 06;13(37.

17. Peres MA, Barbato PR, Reis SCGB, Freitas CHSM, Antunes JLF. Tooth loss in Brazil: analysis of the 2010 Brazilian oral health survey. Rev Saúde Pública 2013 47(Supl 3):1-11.

Recebido em 02/12/2016

Aceito em 27/06/2017
ROSSI K

CERETTA LB

SIMÕES PW

CERETTA RA

RIBEIRO PFA

perfil dos lau -

DOS DE EXAMES

RADIOGRÁFICOS

INTRABUCAIS

REALIZADOS NO

SUL CATARINEN$S E$

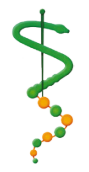

REV, ODONTOL.

UNIV, CID, SÃO

PAULO

2017; 29(2):

110 - 7, MA I - AGO 